\title{
Associated public health and disease consequences of infants/children's exposure to second hand smoking: a systematic review
}

\begin{abstract}
Introduction: An estimated 2.3 million infants/children across all countries of the world are exposed to second-hand smoking (SHS). These infants and children live in households where there is at least one smoker. This has contributed to the alarming incidence of cardiovascular and respiratory diseases, including neuro developmental defects and lowered immunity among infants/children. Illnesses such as asthma, hypertension, arterial defects, impaired neuro development and infectious diseases have been found to be associated with increased exposure to SHS.
\end{abstract}

Method/Methodology: This is a systematic review of published research articles focusing on the health consequences of infants/children's exposure to SHS. Studies reviewed comprise of cross-sectional, prospective, longitudinal and observational studies.

Findings/Results: Key findings indicate that Infants/children are predisposed to various disorders that affect their cardiovascular, respiratory and nervous systems leading to morbidities such as hypertension impaired endothelial functioning, asthma, wheeze, lowered immunity as well as obesity. In addition, permanent disabilities that result in long-term disease conditions such as emphysema, cancer of the lungs and impaired cardiovascular reflex with impaired neuro-development leading to learning disabilities, altered neuro-behaviour as well as anxiety and panic disorders, including depression are all associated with infant/childhood exposure to SHS.

Conclusion: There is immediate public health need to promulgate ways to minimize exposure of infant/childhood to second hand smoking so as to reduce the risks and disease burden associated with SHS.

Keywords: smoking, second hand smoking, infant/children, environmental tobacco, diseases, disabilities, public health
Volume 4 Issue 5 - 2017

\author{
Azuonwu O,' Ominyi JN, ${ }^{2}$ Agom DN² \\ 'Department of Public Health, School of Health, University of \\ Northampton, UK \\ ${ }^{2}$ Department of Nursing, School of Health, University of \\ Northampton, UK
}

Correspondence: Azuonwu O, Department of Public Health, School of Health, University of Northampton, Park Campus, Boughten Green Road, Northhamshire, UK, Tel +234803351986, Emailbimajacobs@yahoo.co.uk

Received: July 16, 2017| Published: September 22, 2017

\section{Introduction}

The World Health Organization (WHO) ${ }^{1}$ reported that about 33\% of male non-smokers, $35 \%$ of female non-smoker and $40 \%$ of children are exposed to passive smoking worldwide. Similarly, the California Environmental Protection Agency (CEPA) ${ }^{2}$ classified passive smoke in conjunction with arsenic, asbestos, benzene and radon as known human carcinogen. Besides, over 4,000 potentially toxic particles and gaseous chemicals are contained in tobacco smoke. ${ }^{3}$ Poisonous chemicals and gases contained in tobacco smoke include nicotine, tar, benzene, carbon (11) oxide, ammonia, formaldehyde and hydrogen cyanide. ${ }^{2}$ However, CEPA ${ }^{2}$ reported that over 50 -cancer causing chemicals are contained in some of these poisonous substances mentioned above. Childhood exposure to second hand smoke leads to short-term health consequences such as nausea, headache, cough, sore throat and dizziness. ${ }^{3}$

In addition, exposure to tobacco smoke increases lung function in adults leading to asthmatic attacks among suffers while it may be induced in children who are predisposed and exposed to parental tobacco smoke. ${ }^{4}$ Similarly, measurable short-term effects such as increased blood pressure, increased bronchial-dilation may also be posed on children who are exposed to second hand smoke by their parents. ${ }^{5}$ Long-term health effects reported include lung cancer, ischemia, chronic obstructive pulmonary disease, otitis media and asthma. ${ }^{3}$ This study focuses on extracting current evidence regarding the health effect of second-hand smoking in infants/ children. In essence, this study explored the health impact of secondhand smoking in infants and children through systematic review of published evidence.

\section{Background}

Two forms of second-hand smoking exist; side-stream and mainstream. Side-streaming is the inhalation of smoke emanating from the lighted end of the cigarette while mainstream is the inhalation of smoke exhaled by a smoker. ${ }^{6}$ Andreas et al., ${ }^{5}$ noted that the combination of both the smoke exhaled by a smoker and the smoke originating from the tip of a flaming cigarette is regarded as passive smoking. Available evidence shows that about $85 \%$ of second-hand smoke is inhaled side-stream while the remaining $15 \%$ goes to the mainstream. ${ }^{7}$ This is because the side-stream contains several millions of semi-liquid particles per $\mathrm{cm}^{3}$ in a mixture of combustive gases. ${ }^{7}$ A mixture of numerous toxins contained in second-hand smoking which are transferable as identified through chemical analysis includes nicotine and other carcinogens which are produced mostly during burning processes associated with smoking. ${ }^{4}$ Despite the fact that both forms of smoking (side and main-stream) are composed of similar chemicals, research evidence indicates that higher percentages of toxins are associated with side-stream second-hand smoking than mainstream. ${ }^{5}$ A reason for this has been attributed to the incomplete combustion and lower temperature associated with lighted cigarette. 
The WHO has labelled second-hand smoking hazardous and a public health issue due to the toxicity accrued from the carcinogens generated by the combustion process. ${ }^{1}$ Several legislations have been proposed by the $\mathrm{WHO}$ all over the world concerning public smoking. ${ }^{1}$ Notably, these legislations have been extended to residential homes and playgrounds where children spend most of their time. Besides, infants and children are part of the most vulnerable section of the world's population according to literature particularly with illnesses related to second-hand smoking. ${ }^{4}$ Available evidence suggests that this is due to the high aeration rate resulting from their low body weight and underdeveloped cells/organs. ${ }^{8}$ Moreover, infants and children are faced with restriction or lack of control in their inability to avoid being exposed and due to the fact that significant cases of second-hand smoking are encountered from carers or parents in their respective homes. ${ }^{5}$

\section{Methods}

\section{Study approach}

The approach adopted for this study was systematic review of selected and published articles. This followed the sequence of systematic review outlined by Aveyard ${ }^{9}$ who defined systematic review as an approach whereby explicit summary of research evidences that are valid and applicable to other studies are extracted and presented following the critical appraisal of a given body of knowledge. The retrieved articles were appraised and presented following extraction of their findings.

\section{Search strategy and search terms}

Different key words were combined while searching the different databases. The following electronic databases were searched; AMED, ASSIA, CINAHL, MEDLINE, PsycNet, Web of Science, Science Direct, SAGE, and Pub Med. Initially, a quick search was conducted on Google to elicit different ways and key words used in describing the topic. In searching through Google researchers ascertain global view about the topic which allows for identification of key words related to the topic area [10]. Abstract of each of the article retrieved was read to ascertain the focus and determine its relevance to the topic under review as well as identify the various ways in which keywords were combined during literature search. The use of Boolean operators and truncations were adopted during the literature search. Key words such as second hand smoking, passive smoking, infant and children were combined in various ways while searching through the databases. Others include health effect, smoke, smoking, smokers as well as disease/illness was equally used to search for articles. The use of 'AND' and 'OR' was also adopted in combining various keywords as it enhances it help to enhance the number of hits generated in a search.

\section{Inclusion criteria}

Peer reviewed published primary research articles which aimed at exploring the health effect of second hand smoking which were published in English language and focusing on infants and children were included in this review. Meanwhile, articles that did not meet these criteria were excluded from this review.

\section{Data extraction and methodological quality of the included studies}

The methodological qualities of the articles included were considered based on the following criteria; sample size, sampling techniques, design frameworks, validity, reliability and generalizability of their findings. Studies were individually appraised and findings presented.

\section{Results}

Following the search on electronic databases, fifteen published articles were retrieved, appraised/reviewed and findings collated. Following the coding of key words extracted from the study findings, four themes were generated. They include effects of second-hand smoking on the respiratory system of infants/children, Effects of second-hand smoking on the cardiovascular system of infants/ children, Effects of second-hand smoking on the infants/children's neuro-development as well as lowered immunity to diseases and infections.

\section{Infant/children's lungs development and growth}

The relationship between second hand smoking and impaired respiratory functioning in infants/children has been demonstrated by the various studies reviewed. Findings show that exposure to second hand smoking in infancy and childhood is strongly associated with respiratory disorders. As reported by Hedman et al., ${ }^{11}$ and Lovasi et al., ${ }^{12}$ pre and post-natal exposure to second hand smoking will not only cause respiratory disorders e.g. asthma and wheeze in children but can also result in long-term disorders such as emphysema and lung cancer. The likelihood of increased chance of respiratory allergy among infants/children's exposed to second hand smoking, ${ }^{3}$ has also been noted. Besides, there is a significant association between infants/children's exposure to second hand smoking and bronchitis as well as hay fever. ${ }^{14}$ Maternal exposure in form of intra-uterine exposure to second hand smoking has also been reported to be strongly associated with asthma diagnosis in childhood. ${ }^{4}$ Lovasi et al., ${ }^{12}$ noted that maternal smoking affects the developmental processes of infants' respiratory systems. These effects are noted to manifest in form of immature airways and respiratory muscles, including the blood vessels. ${ }^{12}$ There is somatic growth inhibition in infants/children who are prenatally exposed to maternal smoking which later results in reduced airflow. ${ }^{14}$.

\section{The cardiovascular system}

Research evidence has implicated cardiovascular diseases as the leading cause of death and disabilities across the world. ${ }^{15}$. Findings have helped in clarifying the harmful effects of childhood exposure to second hand smoking and its relationship with increasing morbidity and disabilities among infants/children. Evidence affirms that maternal exposure to second hand smoking can result in hypertension at later stage in life. ${ }^{15,16}$ High risk of congenital heart defects experienced at infancy has been attributed to maternal exposure to smoking. ${ }^{17}$ This is in addition to impairment in the structure of endothelium and endothelial function which further highlight the harmful nature of childhood exposure to second hand smoking. ${ }^{18}$ Therefore, infant/ childhood exposure to second hand smoking can result in permanent damage to the cardiovascular systems. ${ }^{15-18}$ Nevertheless, the rate of exposure that could result in cardiovascular damage was not specified in the studies reviewed.

\section{Neuro-development and behaviour}

A range of disorders associated with exposure to second hand smoking have been shown to affect infants/children's neuro-behaviour and neuro-development. Maternal smoking during pregnancy exposes 
infants/children to alteration in their neuro-developmental processes and has been described as the harmful effect of drug in the world. ${ }^{19}$. Prenatal tobacco exposure can lead to several environmental hazards that can result in disease and disabilities among infant/children particularly their neuro-behavioural aspect of life. ${ }^{20}$ Stroud et al., ${ }^{19}$ Yolton et al.,$^{20}$ and Lee et al., ${ }^{21}$ highlighted the neuro-developmental effect associated with infants/children's exposure to second hand smoking. Available evidence shows that both pre and post-natal exposure to tobacco smoking can result in altered neuro development and behaviours among infants/children. ${ }^{20}$ Similarly, the risk of developmental delay is significantly associated with exposure to second hand smoking during pregnancy, while infants of non-smoking mothers who are exposed to second hand smoking are at higher risk of experiencing poor neuro-developmental progression. ${ }^{21}$

\section{The immune system and exposure to cigarette smoking}

Association between second hand smoking and increased susceptibility to infection or lowered immunity in infants/children has been documented. Several studies ${ }^{22-25}$ explored the relationship between infants/children's exposure to second hand smoking and their susceptibility to infection. Available evidence suggests that second hand smoking can lead to diseases and disabilities affecting the cardiovascular, respiratory and nervous systems particularly that of the infants/children. ${ }^{23,24}$ Exposure of infants/children to cigarette smoking impairs the development and functioning of the immune system. ${ }^{22}$ Exposure to passive smoking predisposes infants and children to lowered resistant immune system and increases their susceptibility to infections such as gastroenteritis, ${ }^{22,23}$ while periodontal illnesses and Mycobacterium Tuberculosis are prevalent in children who are exposed to second hand smoking. ${ }^{24,26}$

\section{Discussion}

Second hand smoking has long been noted to cause, atherosclerotic and cardiovascular impairments ${ }^{26}$ but recent evidence suggests that infant/childhood exposure to environmental tobacco smoke may equally result in harmful respiratory and neuro-developmental effects with massive public health problems. ${ }^{27}$ This study reveals that respiratory dysfunction is an important early symptom of infant/ childhood exposure to environmental tobacco smoking. Impairment endothelial dilatation is noted to be dose-dependent and may be equivalent to the extent of vascular abnormality found in older adults who engage in active smoking. Exposure to maternal smoking is risk factor for asthma and wheezes, ${ }^{16}$ while both maternal exposure and personal smoking has been independently implicated in asthma and wheeze diagnosis among teenagers. ${ }^{25}$

Maternal exposure is a strong risk factor for physician asthma diagnosis and personal smoking was stronger risk factors for wheeze..$^{25}$ Regarding the clinical outcomes of second hand smoking in infants/ children, available evidence reports adverse consequences on exposed infants/children. There is inverse relationship between endothelial functioning and cotinine (cotinine is a metabolite of nicotine and also a biomarker for the detection of tobacco level in the urine and blood of smokers) levels resulting in lowered production of nitric oxide by the endothelium was reported by Stroud et al., ${ }^{19}$ Meanwhile, there is reduction in arterial wall dilatation following insufficient production of nitric oxide in infants/children. ${ }^{16}$ In addition, increase in the tunica intima media thickness in exposed infants/children results in reduced blood flow as observed in 2401 children earlier studied by Stroud et al. ${ }^{19}$
Besides, in assessing the relationship between infants/children's exposure to second hand smoking and their susceptibility to infection, Hedman et al., ${ }^{11}$ found negative effects of irritants on the mucosal clearance. This is implies that increased bronchial activity may result in lowered mucosal clearance and subsequent exposure to asthma and wheeze as found by Hedman et al. ${ }^{11}$ However, this finding reflects the mechanism through which the infants/children's respiratory system can be affected by these carcinogens. Similarly, Kabir et al., ${ }^{13}$ found that there is increased likelihood of respiratory disorders due to exposure to second hand smoking. Moreover, alterations in the serum level of protein, plasma, lipoprotein, and leptin as well as fibrinogen can result in adverse health outcomes as reported by Cohen et al. ${ }^{15}$ However, increased blood pressure may be due to the presence of circulating cell forming atheromatic plaque in infants/children's. ${ }^{18}$ Moreover, there is higher likelihood of being born with congenital heart defects by mothers who smoke while pregnant despite demographic features and other associated risk factors as observed in Sullivan et al., ${ }^{17}$ study. This is because there is significant association between maternal cigarette use and pulmonary vascular anomalies with associated respiratory disorders. However, stronger risk for cardiovascular anomalies was linked with higher dose of smoking among the subjects studied.

Regarding the effects of exposure to second hand smoking on the infant/children's immune system, Kum-Nji et al., ${ }^{23}$ reported that there is significant low production of interferon- $\gamma$ (INF- $\gamma$ ) in the lungs. INF- $\gamma$ plays a major role in the production of cytokine while cytokine is known to boost the body's immune system to fight invading foreign bodies or infection. ${ }^{23}$ However, Erdemir et al., ${ }^{24}$ documented that a reduction in the production of INF- $\gamma$ increases the susceptibility of infants/children to infection and predisposes them to multiple disorders. As observed in Erdemir et al., ${ }^{24}$ and Kum-Nji et al., ${ }^{23}$ studies, infectious gastroenteritis as well as adverse periodontal health is the health consequences of decreased immunity or increased susceptibility. Besides, reviewed studies reveal that in infancy/ childhood there is underdeveloped immune system which may be suggestive of the likelihood of infants/children to suffer more of the health effects, if exposed to second hand smoking. Furthermore, as observed in the study by Lee et al., ${ }^{21}$ sustained elevation in the level of cardio metabolic biomarkers of the infants with corresponding reduction in the circulating INF- $\gamma$ will result in subsequent increase in the wear and tear of myelin sheath present in the nervous system. The implication of this however, is inflammation of the myelin sheath which can sometimes lead to a disease condition termed multiple sclerosis usually prevalent in children who are exposed to second hand smoking. ${ }^{23}$

More so, concerning the prolonged clinical outcomes of passive smoking, some studies reviewed reported that one of the major risk factors for long-term disorders in adulthood is childhood exposure to second hand smoking. Evidence for this assertion was observed in the Cohen et al. ${ }^{15}$ who highlighted that sustained reduction in cardiac autonomic function is associated with adverse effect of nicotine in increasing the concentration of catecholamine in the blood which results in long-term impairment of cardiovascular reflex action. In the study by Kum-Nji et al., ${ }^{23}$ it was observed that impairment in the normal function of vascular endothelium may be due to elevation in the transfer of endothelial monocyte-like cells which often result in increased adhesion of white blood cells with the vascular endothelium of the exposed infants/children. This might have explained the findings of Gall et al. ${ }^{16}$ study that reported an impairment in the endothelial function of the 2401 children studied which was attributed to lowered 
production of serum nitric oxide. Nonetheless, Ronmark et al., ${ }^{14}$ noted that INF- $\gamma$ can lead to impaired lung functions as observed in the study by Lovasi et al. ${ }^{12}$ who reported that the long-term occurrence of disorders such as lung cancer and emphysema may be due to prolonged reduction in the production of INF- $\gamma$. Besides, increased hyperactivity of the bronchi observed in 3430 participants used in Hedman et al., ${ }^{25}$ study was attributed to lowered production of INF- $\gamma$.

More so, studies regarding the outcomes of childhood exposure to second hand smoking on the infants/children's neuro-development or neuro-behaviour have reported possible relationship between passive smoking and neuro-developmental anomalies in children. However, considering the contrast nature of findings from the studies reviewed in this section, caution must be applied in interpreting their study results. Kabir et al., ${ }^{13}$ tend to associate serum cotinine level with race as they concluded that higher level of serum cotinine was noted among the non-Hispanic black and white children when compared with the Hispanic group. Yolton et al., ${ }^{20}$ study associated the elevated level of serum cotinine to gender; concluding that elevation in serum cotinine level was only observed in the non-Hispanic white boys who had impaired neuro-behaviour. Lee et al., ${ }^{21}$ study asserted that elevation in serum cotinine level depicts higher tendency for impaired neuro-behaviour but concluded that the serum cotinine level varies with race. Perhaps the disparities regarding the findings of the studies relating to relationship between childhood exposure to second hand smoking and neuro-development is an indication of gap in the research which needs to be addressed, particularly on the cotinine metabolism and racial differences.

\section{Conclusion}

Having considered the various findings noted in this review as shown in the discussion, the study hereby conclude that infants/ children's exposure to second hand smoking can result in the following immediate and long-term effects; infants/children are predisposed to various disorders that affect their cardiovascular, respiratory and nervous systems leading to morbidities such as hypertension impaired endothelial functioning, asthma, wheeze, lowered immunity as well obesity; permanent disabilities resulting to long-term disease conditions such as emphysema, cancer of the lungs and impaired cardiovascular reflex; impaired neuro-development leading to learning disabilities, altered neuro-behaviour as well as anxiety and panic disorders, including depression are all associated with infant/ childhood exposure to second hand smoking. Available evidence suggests that parents/carers should be made to stop smoking at home particularly when the children are around. Besides, implementation of initiatives targeted at controlling cigarette/tobacco smoking such as awareness campaign through mass media, explicit labelling of health warning on tobacco packs, increase in prices of tobacco/cigarette and above promulgation of smoke-free policies/legislation particularly in cars and residential homes can alleviate this burden. ${ }^{28}$

\section{Acknowledgements}

None

\section{Conflicts of interest}

There was no observed conflict of interest among authors.

\section{Funding}

None.

\section{References}

1. WHO. Why is smoking an issue for non-smokers. World Health Organisation, Switzerland; 2005.

2. California Environmental Protection Agenc. Secondhand smoke in cars factsheet. 2010.

3. Action on Smoking and Health. Secondhand smoke: the impact on children. ASH Research Report, London, UK; 2011.

4. Jarvie JA, Malone RE. Children's second hand smoking Exposure in Private Homes and Cars: An Ethical Analysis. Health Policy and Ethics. 2008;98(12):2140-2145.

5. Andreas DF, Constantine IV, Giorgos SM, et al. Biological evidence for the acute effect of secondhand smoke exposure. American Journal of Physiology. 2009;298(1):L3-L12.

6. Flouris AD, Metsios G, Carrillo AE, et al. Acute and Short-term Effects of Secondhand Smoke on Lung Function and Cytokine Production. Am J Respir Crit Care Med. 2009;179(11):1029-1033.

7. Tager I. The effects of second- hand and direct exposure to tobacco smoke on asthma and lung function in adolescence. Paediatr Respir Rev. 2008;9(1):29-37.

8. Royal College of Physicians. Passive smoking and children. A report by the Tobacco Advisory, London, UK; 2010. p. 1-207.

9. Aveyard H. Doing a literature review in health and social care. 2nd ed. Open University Press, Maidenhead, UK; 2010.

10. Tod A, Palfreyman S, Burke L. Evidence-based practice is a time of opportunity for nursing. British Journal of Nursing. 2004;13(4):211216.

11. Hedman L, Bjerg A, Sundberg S, et al. Both environmental tobacco smoke and personal smoking is related to asthma and wheeze in teenagers. Thorax. 2011;66(1):20-25.

12. Lovasi GN, Roux AV, Hoffman EA, et al. Association of environmental tobacco smoke exposure in childhood with early emphysema in adulthood among nonsmokers. American Journal of Epidemiology. 2009;171(1):54-62.

13. Kabir Z, Connolly G, Alpert HR. Secondhand smoke exposure and neurobehavioral disorders among children in the United States. Paediatrics. 2011;128(2):263-270.

14. Ronmark E, Ekerljung L, Larsson K, et al. No further increase of incidence of asthma: Incidence, remission and relapse of adult asthma in Sweden. Respiratory Medicine. 2008;102(12):1730-1736.

15. Cohen G, Jeffery H, Lagercrantz H, et al. Long-term reprogramming of cardiovascular functions in infants of active smokers. Journal of the American Heart Association. 2010;55(3):722-728.

16. Gall S, Huynh QL, Magnussen CG, et al. Exposure to parental smoking in childhood or adolescence is associated with increased carotid intimamedia thickness in young adults: evidence from the cardiovascular risk in young Finns study and childhood determinants of adult health study. European Heart Journal. 2014;5(36):2484-2491.

17. Sullivan PM, Dervan LA, Reiger S, et al. Risk of congenital Heart Defects in the offspring of smoking mothers: a population-based study. The Journal of Paediatrics. 2015;166(4):978-984.

18. Geert CC, Bots ML, Van der CK, et al. Parental smoking and vascular damage in their 5-year old children. Paediatrics. 2011;129(1):45-54.

19. Stroud LR, Paster LR, George DP, et al. Maternal smoking during pregnancy and Newborn Neur-behaviour: Effects at 10 to 27 days. Journal of Paediatrics. 2009;154(1):10-16. 
20. Yolton K, Khoury J, Xu Y, et al. Low-level prenatal exposure to nicotine and infant neurobehavior. Neurotoxicol Teratol. 2009;31(6):356-363.

21. Lee B, Hong Y, Park H, et al. Second hand smoke exposure during pregnancy and infantile neuro-development. Environmental Research. 2011;111(4):539-544

22. Muhammad H, Hoek G, Luttman-Gibson H, et al. Parental smoking and lung function in children; an international study. American Journal of Respiratory Critical Care Medicine. 2006;73(11):1255-1263.

23. Kum-Nji P, Mangrem LC, Wells PJ, et al. Is environmental tobacco smoke exposure a risk factor for acute gastroenteritis in young children? Pediatrics. 2009;48(7):756-762.

24. Erdemir EO, Sonmez IS, Oba AA, et al. Periodontal health in children exposed to passive smoking. Journal of Clinical Periodontal. 2010;37(2):160-164.
25. Den Boon S, Verver S, Lombard CJ, et al. Comparison of symptoms and treatment outcomes between actively and passively detected tuberculosis cases: the additional value of active case finding. Epidemiology and Infection. 2007;136(10):1342-1348.

26. Bélanger M, O’Loughlin J, Okoli CT, et al. Nicotine dependence symptoms among young never-smokers exposed to secondhand tobacco smoke. Addictive Behaviors. 2008;33(12):1557-1563.

27. AL-Rawas OA, AL-Maniri AA, AL-Riyami BM. Home exposure to Arabian incense (bakhour) and asthma symptoms in children: a community survey in two regions in Oman. BMC pulmonary medicine. 2009;9:23.

28. Öberg M, Jaakkola MS, Woodward A, et al. Worldwide burden of disease from exposure to second-hand smoke: a retrospective analysis of data from 192 countries. The Lancet. 2011;377(9760):139-146. 